\title{
A Blind Two-Stage Receiver for MIMO-CDMA Systems
}

\author{
Fuh-Hsin Hwang ${ }^{1}$ and Tsui-Tsai Lin ${ }^{2}$ \\ ${ }^{1}$ Department of Optoelectronics and Communication Engineering, National Kaohsiung Normal University, 62 Shenjhong Road, \\ Yanchao Township, Kaohsiung County 824, Taiwan \\ ${ }^{2}$ Department of Electronic Engineering, National United University, 1 Lien-Da, Kung-Ching Li, Miaoli 360, Taiwan
}

Correspondence should be addressed to Tsui-Tsai Lin, ttlincs@nuu.edu.tw

Received 23 February 2011; Accepted 24 March 2011

Academic Editors: H. Ikeda, A. Maaref, and H.-L. Wei

Copyright ( $) 2011$ F.-H. Hwang and T.-T. Lin. This is an open access article distributed under the Creative Commons Attribution License, which permits unrestricted use, distribution, and reproduction in any medium, provided the original work is properly cited.

\begin{abstract}
A blind two-stage multiple-input multiple-output (MIMO) receiver is proposed for code-division multiple access (CDMA) systems utilizing space-time block coding (STBC) over a multipath fading channel. Specifically, in the first stage, a signal-blocked (SB) interference-blocked (IB) detector is first constructed for collection of the desired signals and suppression of multiple access interference (MAI). In the second-stage, a decision-directed scheme is developed to alleviate desired signal cancellation. Computer simulations demonstrate that the proposed blind receiver can achieve a reliable output signal-to-interference-plus-noise ratio (SINR) performance approximating that of the optimal minimum mean squared error (MMSE) receiver and can exhibit the robustness against the finite data sample effect.
\end{abstract}

\section{Introduction}

Code-division multiple access (CDMA) with the advantages of high transmission rates and capacity potential, and an intrinsic resistance to narrowband interference have been the leading solutions and technical standard specifications for the wireless network applications [1]. In spite of these advantages, however, signals from other users, referred to as multiple access interference (MAI), with higher transmission power caused by the near-far problem cannot be efficiently suppressed because of insufficient processing gains. This leads to a substantial degradation in performance.

The multiple-input multiple-output (MIMO) system [24] using the space-time block coding (STBC) scheme [3] to provide spatial diversity can promise an enormous increase in system capacity and link quality without sacrificing the radio frequency resource. Recently, CDMA systems employing the MIMO and STBC techniques have been proposed and discussed to support the tremendous growth in various highrate services. The physical layer impediments in the design and implementation of the CDMA MIMO receiver are MAI and channel dispersion impairment. Especially for the STBC system, the MAI resulting from the interference between antennas is more severe than that in single-antenna systems.
The minimum mean squared error (MMSE) receiver has been extensively discussed and used as a remedy to combat MAI [2]. However, its performance is sensitive to both the mismatches between the true and user channel state information (CSI) matrix and the finite sample effect. In order to correctly estimate the CSI matrix, a larger amount of preknown pilot symbols is required and leads to a significant decrease in the system throughput [5]. As an alternative, a nondata aided signal-blocked (SB) interference-blocked (IB) receiver [6] was proposed for direct sequence CDMA system. It has been shown that the SBIB receiver can successfully collect the desired signal and suppress MAI for a singleinput single-output (SISO) system over a multipath fading channel. Partially adaptive (PA) filtering [7], which uses a portion of the degree of freedom available in the weight adaptation process, is a computational efficient scheme for various signal processing applications. The subspace-based two-stage receivers [8] adopt the generalized sidelobe canceller (GSC) incorporating with the PA algorithm to improve performance of channel estimation and symbol detection for Alamouti's STBC CDMA MIMO system over a one-path fading channel.

In this paper, over a multipath fading channel, we proposed a blind two-stage MIMO receiver that can 
achieve a similar output signal-to-interference-plus-noise ratio (SINR) as the optimal MMSE receiver but got rid of requirement of the preknown pilot symbols. Specifically, the design of the proposed scheme involves the following procedure. In the first stage, an SB transformation is first developed before processing MAI suppression for circumventing desired signal cancellation. An IB transformation is then constructed based on the SB transformed data and is used to suppress the MAI blindly. Finally, the desired signals buried in the IB transformed data are combined in accordance with maximizing the output signal-to-noise ratio (SNR) criterion. In the second stage, the subspace MMSE (SMMSE) receiver [9] incorporating with a decision-directed scheme is developed to mitigate desired signal cancellation resulting from the finite sample effect. Simulation results indicate that the proposed receiver exhibits significant performance improvement when compared with conventional methods.

The notations used in this paper follow that the usual convention-bold capital letters denote vectors or matrices, $\mathbf{I}_{n}$ is an $n \times n$ identity matrix, $\mathbf{0}_{n}$ is an all-zero vector of size $n \times 1, \mathbf{O}_{m \times n}$ is an all-zero matrix of size $m \times n, \odot$ is the Kronecker product, and $(\cdot)^{*},(\cdot)^{T}$, and $(\cdot)^{H}$ are complex conjugate, transpose, and Hermitian transpose of $(\cdot)$, respectively. We also use $E\{\cdot\}$ to denote ensemble average, $|\cdot|$ for absolute operator, ||$\cdot \|$ for Euclidean norm, and $\operatorname{tr}\{\cdot\}$ for the trace operator. $\arg \{\cdot\}$ and $\operatorname{Arg}\{\cdot\}[10]$ denote an argument and the argument of a complex number, respectively. Finally, the operators $\bmod \{\cdot\}$ and $\operatorname{dec}\{\cdot\}$ stand for computing the modulus after division and the data decision operator, respectively.

\section{Data Model}

Consider herein a $K$-user CDMA system equipped with two transmit antennas (TXs) and $N$ receive antennas (RXs) over a multipath fading channel with $L$-resolvable paths, in which the Alamouti's STBC scheme is adopted to provide transmit diversity. Each modulated signal of $k$ th user is spread with a unique spreading code $\mathbf{c}_{k}^{(1)}\left(\mathbf{c}_{k}^{(2)}\right)$ of length $M$ for TX1 (TX2) [4]. After spreading, a zero-padding of $N_{g}$ samples, assumed to be longer than the expected maximum excess delay $L$, is added to combat intersymbol interference (ISI) caused by multipath. Finally, the resultant data is fed into the multiple access channel. At the receiver end, the received signal is chip matched filtered, sampled at the chip rate over the entire observation interval of chips, and then punctured the $N_{g}-$ $L+1$ message-free samples. According to the Alamouti's STBC scheme utilizing two transmit antennas, the $P \times 1$ received data vectors, where $P=M+L-1$, corresponding to the $n$th receive antenna over two consecutive symbol intervals are written by [4]

$$
\begin{gathered}
\mathbf{x}_{n}(2 i-1)=\sum_{k=1}^{K}\left[\mathbf{h}_{n, k}^{(1)} d_{k}(2 i-1)+\mathbf{h}_{n, k}^{(2)} d_{k}(2 i)\right]+\mathbf{n}_{n}(2 i-1), \\
\mathbf{x}_{n}(2 i)=\sum_{k=1}^{K}\left[-\mathbf{h}_{n, k}^{(1)} d_{k}^{*}(2 i)+\mathbf{h}_{n, k}^{(2)} d_{k}^{*}(2 i-1)\right]+\mathbf{n}_{n}(2 i),
\end{gathered}
$$

where $d_{k}(i)$ is the $i$ th transmitted symbol of user $k$ assumed to be independent and identically distributed (i.i.d.) with zero-mean and unit variance, and the vector $\mathbf{n}_{n}(i)$ is the spatially and temporally white noise of the covariance matrix $\sigma_{n}^{2} \mathbf{I}_{P}$, with $\sigma_{n}^{2}$ being the noise power. Finally, the $P \times 1$ vector $\mathbf{h}_{n, k}^{(m)}$ denotes the effective composite signature vector (CSV) of user $k$ from TXm to RX $n$ given by

$$
\mathbf{h}_{n, k}^{(m)}=\sigma_{k} \sum_{l=1}^{L} g_{n, l}^{(m)} \mathbf{c}_{k, l}^{(m)}=\sigma_{k} \mathbf{C}_{k}^{(m)} \mathbf{g}_{n}^{(m)}, \quad m=1,2,
$$

where

$$
\mathbf{C}_{k}^{(m)}=\left[\mathbf{c}_{k, 1}^{(m)}, \mathbf{c}_{k, 2}^{(m)}, \ldots, \mathbf{c}_{k, L}^{(m)}\right], \quad \mathbf{g}_{n}^{(m)}=\left[g_{n, 1}^{(m)}, g_{n, 2}^{(m)}, \ldots, g_{n, L}^{(m)}\right]^{T} .
$$

The scalar $\sigma_{k}^{2}$ is the transmit power of the $k$ th user, $g_{n, l}^{(m)}$ is the complex channel gain from TXm to RX $n$ of the $l$ th path, and $\mathbf{c}_{k, l}^{(m)}=\left[\mathbf{0}_{l-1}^{T}, \mathbf{c}_{k}^{(m) T}, \mathbf{0}_{L-l}^{T}\right]^{T}$ is the augmented signature vector associated with the $l$ th path of user $k$. According to the STBC structure and stacking the two consecutive received data samples in (1), we have

$$
\mathbf{y}_{n}(i)=\left[\begin{array}{c}
\mathbf{x}_{n}(2 i-1) \\
\mathbf{x}_{n}^{*}(2 i)
\end{array}\right]=\sum_{k=1}^{K} \mathbf{H}_{n, k} \mathbf{d}_{k}(i)+\mathbf{v}_{n}(i),
$$

where

$$
\begin{gathered}
\mathbf{H}_{n, k}=\left[\begin{array}{cc}
\mathbf{h}_{n, k}^{(1)} & \mathbf{h}_{n, k}^{(2)} \\
\mathbf{h}_{n, k}^{(2)^{*}} & -\mathbf{h}_{n, k}^{(1)^{*}}
\end{array}\right], \quad \mathbf{d}_{k}(i)=\left[\begin{array}{c}
d_{k}(2 i-1) \\
d_{k}(2 i)
\end{array}\right], \\
\mathbf{v}_{n}(i)=\left[\begin{array}{c}
\mathbf{n}_{n}(2 i-1) \\
\mathbf{n}_{n}^{*}(2 i)
\end{array}\right] .
\end{gathered}
$$

Without loss of generality, we assume that the first user is the desired user. The received array data vector can be expressed as

$$
\mathbf{y}(i)=\left[\mathbf{y}_{1}^{T}(i), \mathbf{y}_{2}^{T}(i), \ldots, \mathbf{y}_{N}^{T}(i)\right]^{T}=\sum_{k=1}^{K} \mathbf{H}_{k} \mathbf{d}_{k}(i)+\mathbf{v}(i),
$$

where

$$
\begin{gathered}
\mathbf{H}_{k}=\left[\mathbf{H}_{1, k}^{T}, \mathbf{H}_{2, k}^{T}, \ldots, \mathbf{H}_{N, k}^{T}\right]^{T}, \\
\mathbf{v}(i)=\left[\mathbf{v}_{1}^{T}(i), \mathbf{v}_{2}^{T}(i), \ldots, \mathbf{v}_{N}^{T}(i)\right]^{T} .
\end{gathered}
$$

A linear receiver is designed a $2 \mathrm{NP} \times 2$ weight matrix $\mathbf{W}_{1}$ to extract and remove $\mathbf{H}_{1}$ to retrieve the transmitted data $\mathbf{d}_{1}(i)$ from the received signal. Among them, the MMSE receiver [2], which is widely and popularly employed for CDMA systems, minimizes the mean squared error (MSE) between the receiver output and known pilot symbols $\mathbf{d}_{1}(i)$ :

$$
\begin{aligned}
& \min _{\mathbf{W}_{1}} E\left\{\left\|\mathbf{W}_{1}^{H} \mathbf{y}(i)-\mathbf{d}_{1}(i)\right\|^{2}\right\} \\
& \equiv \min _{\mathbf{W}_{1}} \mathbf{W}_{1}^{H} \mathbf{R} \mathbf{W}_{1}-\mathbf{W}_{1}^{H} \mathbf{H}_{1}-\mathbf{H}_{1}^{H} \mathbf{W}_{1}+\mathbf{I}_{2},
\end{aligned}
$$


whose solution is given by

$$
\mathbf{W}_{1}=\mathbf{R}^{-1} \mathbf{H}_{1},
$$

where $\mathbf{R}$ is the data correlation matrix defined by

$$
\mathbf{R}=E\left\{\mathbf{y}(i) \mathbf{y}^{H}(i)\right\}=\sum_{k=1}^{K} \mathbf{H}_{k} \mathbf{H}_{k}^{H}+\sigma_{n}^{2} \mathbf{I}_{2 \mathrm{NP}}
$$

The MMSE receiver has been shown to successfully detect the transmit data of the desired user and deal with MAI as long as the CSI matrix $\mathbf{H}_{1}$ can be distortionlessly estimated beforehand. However, both the estimation error in $\mathbf{H}_{1}$ and the finite sample effect will induce desired signal cancellation. This significantly drops the system performance. As a remedy, a larger amount of pilot symbols is necessary for the MMSEbased receiver, especially for high input SNR. Unfortunately, in practice, this is impossible due to the limitation of radio frequency resources [5].

\section{Proposed Two-Stage MIMO Receiver}

In this section, the proposed blind two-stage MIMO receiver is developed. In the first stage, the SB and IB transformations are constructed to alleviate desired signal cancellation and to resist interfering signals, respectively. An optimal combiner obtained in accordance with maximizing the output SNR is then performed on the IB transformed data for collecting the path signals. In the second stage, a decision-directed scheme is developed to enhance the robustness against the finite sample effect.

3.1. Signal-Blocked Interference-Blocked Receiver. Conventional MMSE receiver is found to be sensitive to the errors in the CSI estimation, which will cause desired signal cancellation. To avoid such degradation, a $2 \mathrm{NP} \times 2 \mathrm{NP}$ linear transformation matrix $\mathbf{T}_{\mathrm{SB}}$ executing on the received data before processing MAI suppression is designed to remove the desired signal corresponding to $d_{1}(2 i-1)$ :

$$
\begin{array}{r}
\mathbf{T}_{\mathrm{SB}}\left[\mathbf{0}_{2(n-1) P}^{T}, \mathbf{c}_{1, l}^{(1) T}, \mathbf{0}_{[2(N-n)+1] P}^{T}\right]^{T}=\mathbf{0}_{2 \mathrm{NP}}, \\
\mathbf{T}_{\mathrm{SB}}\left[\mathbf{0}_{(2 n-1) P}^{T}, \mathbf{c}_{1, l}^{(2) T}, \mathbf{0}_{2(N-n) P}^{T}\right]^{T}=\mathbf{0}_{2 \mathrm{NP}}, \\
\quad l=1,2, \ldots, L ; n=1,2, \ldots, N,
\end{array}
$$

such that the SB transformed data vector can be expressed by

$$
\mathbf{T}_{\mathrm{SB}} \mathbf{y}(i)=\mathbf{T}_{\mathrm{SB}} \mathbf{H}_{1}(:, 2) d_{1}(2 i)+\mathbf{T}_{\mathrm{SB}} \sum_{k=2}^{K} \mathbf{H}_{k} \mathbf{d}_{k}+\mathbf{T}_{\mathrm{SB}} \mathbf{v}(i)
$$

where $\mathbf{H}_{1}(:, i)$ denotes the $i$ th column of the matrix $\mathbf{H}_{1}$. As expected, the SB transformation can completely remove the desired signal $d_{1}(2 i-1)$ because of preknown spreading codes $\mathbf{c}_{1}^{(m)}$ for $m=1,2$.

Comparing (12) with (6) indicates that, except for the desired signal, the only difference between them is the term involving the self-interference $d_{1}(2 i)$, the uncorrelated interference, and noise. Thus, in order for the weight vector working on the transformed data to suppress the interference, the remaining degree of freedom in $\mathbf{T}_{\mathrm{SB}}$ should be exploited to minimize the error

$$
E\left\{\left\|\mathbf{T}_{\mathrm{SB}} \mathbf{y}(i)-\mathbf{y}(i)\right\|^{2}\right\} \equiv \operatorname{tr}\left\{\left(\mathbf{T}_{\mathrm{SB}}-\mathbf{I}_{2 \mathrm{NP}}\right) \mathbf{R}\left(\mathbf{T}_{\mathrm{SB}}-\mathbf{I}_{2 \mathrm{NP}}\right)^{H}\right\} .
$$

In corporation of the linear constraints of (11) in the minimization of (13) leads the following constrained problem:

$$
\begin{aligned}
& \min _{\mathbf{T}_{\mathrm{SB}}} E\left\{\left\|\mathbf{T}_{\mathrm{SB}} \mathbf{y}(i)-\mathbf{y}(i)\right\|^{2}\right\}, \\
& \text { subject to: } \mathbf{T}_{\mathrm{SB}} \widetilde{\mathbf{C}}=\mathbf{O}_{2 \mathrm{NP} \times 2 \mathrm{LN}},
\end{aligned}
$$

whose solution is given by

$$
\mathbf{T}_{\mathrm{SB}}=\mathbf{I}_{2 \mathrm{NP}}-\tilde{\mathbf{C}}\left(\widetilde{\mathbf{C}}^{H} \mathbf{R}^{-1} \tilde{\mathbf{C}}\right)^{-1} \widetilde{\mathbf{C}} \mathbf{R}^{-1},
$$

where

$$
\widetilde{\mathbf{C}}=\left[\begin{array}{cc}
\mathbf{C}_{1}^{(1)} & \mathbf{O}_{P \times L} \\
\mathbf{O}_{P \times L} & \mathbf{C}_{1}^{(2)}
\end{array}\right] \odot \mathbf{I}_{N}
$$

With the assumption of sufficient degree of freedom in computing $\mathbf{T}_{\mathrm{SB}}$, the $\mathrm{SB}$ transformed data can be approximately expressed by

$$
\mathbf{T}_{\mathrm{SB}} \mathbf{y}(i) \approx \mathbf{H}_{1}(:, 2) d_{1}(2 i)+\sum_{k=2}^{K} \mathbf{H}_{k} \mathbf{d}_{k}+\mathbf{v}(i) .
$$

According to (17), the interference-plus-noise subspace can be extracted from the SB transformed data, chiefly consisting of interference and noise. Under the condition of $(2 K-1)<$ $2 \mathrm{NP}$, the SB transformed data correlation matrix can be eigen-decomposed as

$$
\begin{aligned}
\widetilde{\mathbf{R}} & =E\left\{\mathbf{T}_{\mathrm{SB}} \mathbf{y}(i) \mathbf{y}^{H}(i) \mathbf{T}_{\mathrm{SB}}^{H}\right\} \equiv \mathbf{T}_{\mathrm{SB}} \mathbf{R} \mathbf{T}_{\mathrm{SB}}^{H} \\
& \approx \mathbf{H}_{1}(:, 2) \mathbf{H}_{1}^{H}(:, 2)+\sum_{k=2}^{K} \mathbf{H}_{k} \mathbf{H}_{k}^{H}+\sigma_{n}^{2} \mathbf{I}_{2 \mathrm{NP}} \\
& =\sum_{i=1}^{2 K-1}\left(\lambda_{i}-\sigma_{n}^{2}\right) \mathbf{e}_{i} \mathbf{e}_{i}^{H}+\sigma_{n}^{2} \mathbf{I}_{2 \mathrm{NP}}
\end{aligned}
$$

where $\lambda_{i}$ for $i=1,2, \ldots, 2 K-1$ in the decent order denote the $(2 K-1)$ largest eigenvalues of $\widetilde{R}$ with the corresponding eigenvectors $\mathbf{e}_{i}$ for $i=1,2, \ldots, 2 K-1$. Obviously, the interference part associated with the SB transformed data correlation matrix in (18) can be expressed in terms of the $(2 K-1)$ principal eigenvectors $\mathbf{e}_{i}$ for $i=1,2, \ldots, 2 K-1$. On the other hand, the complement of the interference-plusnoise subspace spanned by $\left\{\mathbf{e}_{1}, \mathbf{e}_{2}, \ldots, \mathbf{e}_{2 K-1}\right\}$ can be used to suppress the interference. Hence, the general solution of the IB transformation $\mathrm{T}_{\mathrm{IB}}$ is suggested by

$$
\mathbf{T}_{\mathrm{IB}}=\mathbf{I}_{2 \mathrm{NP}}-\mathbf{E E}^{H},
$$


where $\mathbf{E}=\left[\mathbf{e}_{1}, \mathbf{e}_{2}, \ldots, \mathbf{e}_{2 K-1}\right]$. Note that $\mathbf{T}_{I B}$ is an idempotent and Hermitian matrix such that $\mathrm{T}_{\mathrm{IB}}^{2}=\mathrm{T}_{\mathrm{IB}}$ and $\mathrm{T}_{\mathrm{IB}}^{H}=\mathrm{T}_{\mathrm{IB}}$. According to (6) and (19), the IB transformed data, containing only the desired signal and noise, can be expressed by

$$
\mathbf{T}_{\mathrm{IB}} \mathbf{y}(i) \approx \mathbf{T}_{\mathrm{IB}} \mathbf{H}_{1}(:, 1) d_{1}(2 i-1)+\mathbf{T}_{\mathrm{IB}} \mathbf{v}(i) .
$$

The weight vector constructed based on the IB transformed data is designed to be capable of constructively combining the desired signal introduced by multipath. To achieve an optimal performance, the corresponding weight vector can be determined in accordance with maximizing the output SNR:

$$
\begin{aligned}
\max _{\mathbf{W}_{1}} \frac{E\left\{\left|\mathbf{W}_{1}^{H}(:, 1) \mathbf{T}_{\mathrm{IB}} \mathbf{H}_{1}(:, 1) d_{1}(2 i-1)\right|^{2}\right\}}{E\left\{\left|\mathbf{W}_{1}^{H}(:, 1) \mathbf{T}_{\mathrm{IB}} \mathbf{v}(i)\right|^{2}\right\}} \\
\equiv \frac{\mathbf{W}_{1}^{H}(:, 1) \mathbf{T}_{\mathrm{IB}} \mathbf{H}_{1}(:, 1) \mathbf{H}_{1}^{H}(:, 1) \mathbf{T}_{\mathrm{IB}} \mathbf{W}_{1}(:, 1)}{\mathbf{W}_{1}^{H}(:, 1) \mathbf{T}_{\mathrm{IB}} \mathbf{W}_{1}(:, 1)} .
\end{aligned}
$$

Unfortunately, $\mathbf{H}_{1}(:, 1)$ in the above equation is not available in practice. Since the MAI can successfully eliminate by the IB transformation, the combiner output power is approximately equal to the signal-plus-noise power. According to the fact that maximizing the signal-plus-noise-to-noise ratio (SNNR) is effective the same as to maximize SNR, (21) can be rewritten as

$$
\max _{\mathbf{W}_{1}} \frac{E\left\{\left|\mathbf{W}_{1}^{H}(:, 1) \mathbf{T}_{\mathrm{IB}} \mathbf{y}(i)\right|^{2}\right\}}{E\left\{\left|\mathbf{W}_{1}^{H}(:, 1) \mathbf{T}_{\mathrm{IB}} \mathbf{v}(i)\right|^{2}\right\}} \equiv \frac{\mathbf{W}_{1}^{H}(:, 1) \mathbf{T}_{\mathrm{IB}} \mathbf{R} \mathbf{T}_{\mathrm{IB}} \mathbf{W}_{1}(:, 1)}{\mathbf{W}_{1}^{H}(:, 1) \mathbf{T}_{\mathrm{IB}} \mathbf{W}_{1}(:, 1)} .
$$

The solution is given by the dominant mode (the eigenvector associated with the largest eigenvalue) of the eigen-equation

$$
\mathbf{T}_{\mathrm{IB}} \mathbf{R T}_{\mathrm{IB}} \mathbf{W}_{1}(:, 1)=\eta \mathbf{T}_{\mathrm{IB}} \mathbf{W}_{1}(:, 1),
$$

where $\eta$ denotes the largest eigenvalue. Ideally, the degradation of output SINR is mainly caused by the components in the complement of the desired signal subspace such that the optimal weight vector formed by projecting the weight vector $\mathbf{W}_{1}(:, 1)$ onto the desired signal-plus-noise subspace (complement of the interference-plus-noise subspace) can be given by [11]

$$
\widetilde{\mathbf{W}}_{1}(:, 1)=\mathbf{T}_{\mathrm{IB}} \mathbf{W}_{1}(:, 1) \text {. }
$$

3.2. Decision-Directed MMSE Receiver. In order to further improve the robustness against the finite data sample effect, we proposed a decision-directed MMSE receiver. For a moderately good condition, the receiver output is approximately expressed by

$$
\begin{aligned}
z_{1}(i) & =\widetilde{\mathbf{W}}_{1}^{H}(:, 1) \mathbf{y}(i) \\
& \approx \widetilde{\mathbf{W}}_{1}^{H}(:, 1) \mathbf{H}_{1}(:, 1) d_{1}(2 i-1)+\widetilde{\mathbf{W}}_{1}^{H}(:, 1) \mathbf{v}(i) .
\end{aligned}
$$

It is interesting to note that the receiver output in (25) and the transmit information data $d_{1}(2 i-1)$ differ by a complex scalar $\widetilde{\mathbf{W}}_{1}^{H}(:, 1) \mathbf{H}_{1}(:, 1)$. With the assumptions of BPSKmodulated signal and the successful suppression of MAI, the phase of $\widetilde{\mathbf{W}}_{1}^{H}(:, 1) \mathbf{H}_{1}(:, 1), \varphi_{1}$, can be obtained based on the receiver output, leading to the following result:

$$
\hat{\varphi}_{1}=\frac{\sum_{i=1}^{N_{s}} \bmod \left\{\arg \left\{z_{1}(i)\right\}, \pi\right\}}{N_{s}} \approx \arg \left\{\widetilde{\mathbf{W}}_{1}^{H}(:, 1) \mathbf{H}_{1}(:, 1)\right\},
$$

where $N_{s}$ is the number of data samples. For high output SINR, the phase estimator in (26) performs well with a small MSE. In the extreme case, as the value of output SINR goes infinite, the proposed scheme is consistent in the mathematical sense [6]. However, an argument of a complex number $\arg \{\cdot\}$ is infeasible in implementation. An alternative approach uses the principal angle $\operatorname{Arg}\{\cdot\}$ instead of $\arg \{\cdot\}$, in which value for $\operatorname{Arg}\{\cdot\}$ lies between $-\pi$ and $\pi$. According to the assumptions of high output SINR and $\widetilde{\mathbf{W}}_{1}^{H}(:, 1) \mathbf{H}_{1}(:, 1)$ in the first or second quadrant, that is, $0 \leq$ $\varphi_{1}<\pi$, we have the principal angle of the receiver output $z_{1}$ [10]:

$$
\operatorname{Arg}\left\{z_{1}\right\}= \begin{cases}\varphi_{1}, & d_{1}(2 i-1)=1 \\ \varphi_{1}-\pi, & d_{1}(2 i-1)=-1 .\end{cases}
$$

Thus, the phase estimate is given by $\hat{\varphi}_{1}=\operatorname{Arg}\left\{\widetilde{\mathbf{W}}_{1}^{H}(\right.$ :, 1) $\left.\mathbf{H}_{1}(:, 1)\right\}$, which means that the phase $\varphi_{1}$ can be correctly obtained by (26). On the contrary, for $-\pi<\varphi_{1}<0$, the output of the argument of the receiver output is $\varphi_{1}$ and $\varphi_{1}+\pi$ for $d_{1}(2 i-1)=1$ and -1 , respectively, such that the phase estimation of the proposed scheme is $\varphi_{1}+\pi$. It is noteworthy that there is a $\pi$-phase ambiguity involving the phase estimate. Without the further assumptions, we cannot resolve the $\pi$-phase ambiguity. Fortunately, in existence of the digital communications systems, a few predetermined bits, for example, the preamble, parity check, synchronization, or reserved bits [12-14], can be used to get rid of this problem. Furthermore, the phase estimator in (26) can be applied to the phase shift keying (PSK) modulated signals with a little modification.

By exploiting the phase estimate in (26), the transmit information symbols can be decoded by a data decision device, given by

$$
\hat{d}_{1}(2 i-1)=\operatorname{dec}\left\{z_{1}(i) e^{-j \hat{\varphi}_{1}}\right\} .
$$

A simple and nature way to obtain $\hat{\mathbf{H}}_{1}(:, 1)$ would be to use $\hat{d}_{1}(2 i-1)$ as pilot symbols [5]:

$$
\hat{\mathbf{H}}_{1}(:, 1)=\frac{\sum_{i=1}^{N_{s}} \mathbf{y}(i) \hat{d}_{1}(2 i-1)}{N_{s}} .
$$

Finally, the CSI estimate in (29) can be incorporated into the S-MMSE scheme [9] to obtain the first column of the weight matrix. Note that, according to the STBC structure, the second column of the weight matrix $\mathbf{W}_{1}$ can be constructed by its first column such that its corresponding derivation is omitted for simplicity [4]. 


\section{Computer Simulations}

Computer simulations were conducted to ascertain the efficacy of the proposed two-stage receiver in a synchronous ten-user $(K=10)$ CDMA system using two receive antennas $(N=2)$. For all users, the signals were spread by random codes of length $M=32$ through a four-path $(L=4)$ Rayleigh fading channel, in which the path delays were uniformly distributed and the path gains were i.i.d. unit variance complex Gaussian random variables. The input SNR and near-far ratio (NFR) were defined as SNR $=10 \times$ $\log _{10}\left(\sigma_{1}^{2} / \sigma_{n}^{2}\right)$ and NFR $=10 \times \log _{10}\left(\sigma_{k}^{2} / \sigma_{1}^{2}\right)$, respectively. All MAI power was assumed to be identical for simplicity. We assumed that the $\pi$-phase ambiguity can be solved by using the preknown data bits [12-14]. In each simulation, a total of 500 independent trials were executed to compute the output SINR values, with each trial using a different set of spreading codes and data/noise sequences. For performance comparison, we included the results obtained with the SMMSE receiver [9] using the true CSI matrix and the timemultiplexed pilot symbol assisted MMSE receiver [5], in which $N_{p}$ pilot symbols were used to estimate the CSI matrix. Note that the fully-known data-aided S-MMSE receiver, that is, $N_{p}=N_{s}$, is referred to as the optimal receiver.

In the first set of simulations, the output SINR performance was evaluated by varying input SNR with NFR $=10 \mathrm{~dB}$ and $N_{s}=500$. The corresponding results shown in Figure 1 indicated that the proposed receiver achieved a similar performance as the optimal one and outperformed the others. This confirmed that the proposed receiver can successfully collect the desired signals and suppress MAI. On the other hand, the pilot symbol assisted receivers cannot correctly estimate the CSI matrix due to strong MAI, which led to a poor performance.

In the second set of simulations, robustness of the proposed two-stage receiver against MAI was investigated as a function of different NFR values with SNR $=-10 \mathrm{~dB}$ and $N_{s}=500$. As shown in Figure 2, the proposed scheme performed like the optimal receiver, which demonstrated an excellent near-far resistance in cancelling strong MAI. It is noteworthy that, in the case of NFR $=0 \mathrm{~dB}$, the proposed receiver produced a slight degradation about $0.28 \mathrm{~dB}$ in output SINR. This is because the weaker MAI put less emphasis on the SB transformation which caused a transformation error. Fortunately, the effect of the weaker MAI can be significantly alleviated by means of the inherent property of the direct-sequence CDMA systems. Thus, this problem can be neglected. In addition, the existence of strong MAI can be determined by the strong interference detector [15].

In the third set of simulations, the convergence behavior was examined by varying the data sample size $N_{s}$ with $\mathrm{SNR}=$ $-10 \mathrm{~dB}$ and NFR $=10 \mathrm{~dB}$. The results given in Figure 3 demonstrated that the proposed scheme with a similar performance as the optimal receiver converged in about $N_{s}=$ 500 data symbols. On the contrary, the other S-MMSE receivers cannot provide a reliable performance even in the case of 3000 data samples because of the finite data sample effect again.

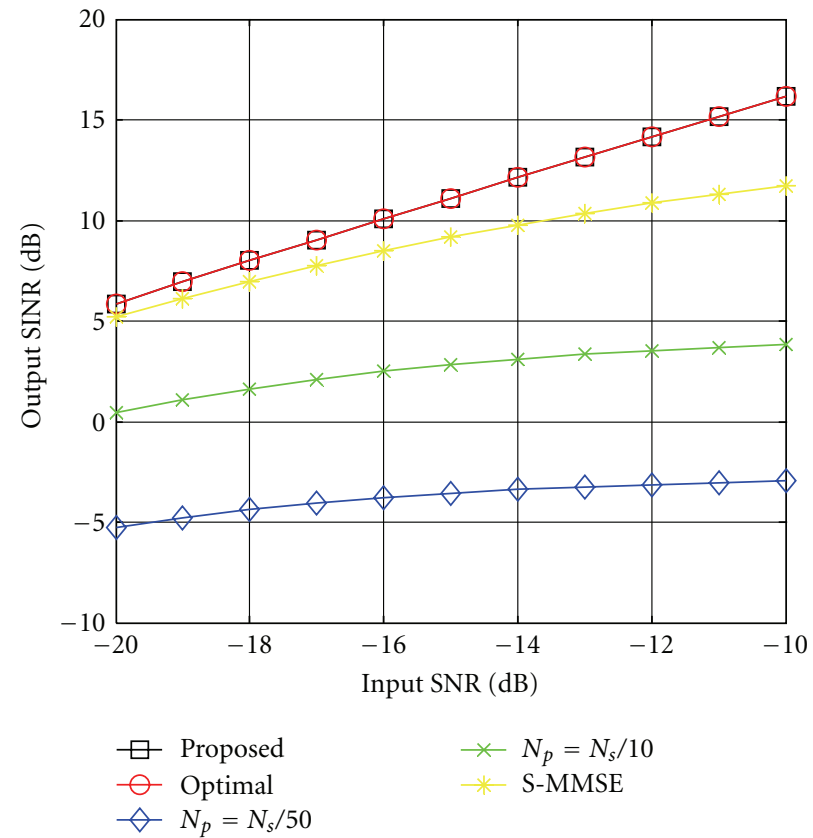

FIGURE 1: Output SINR performance versus input SNR with $N=2$, $M=32, L=4, K=10, \mathrm{NFR}=10 \mathrm{~dB}$, and $N_{s}=500$.

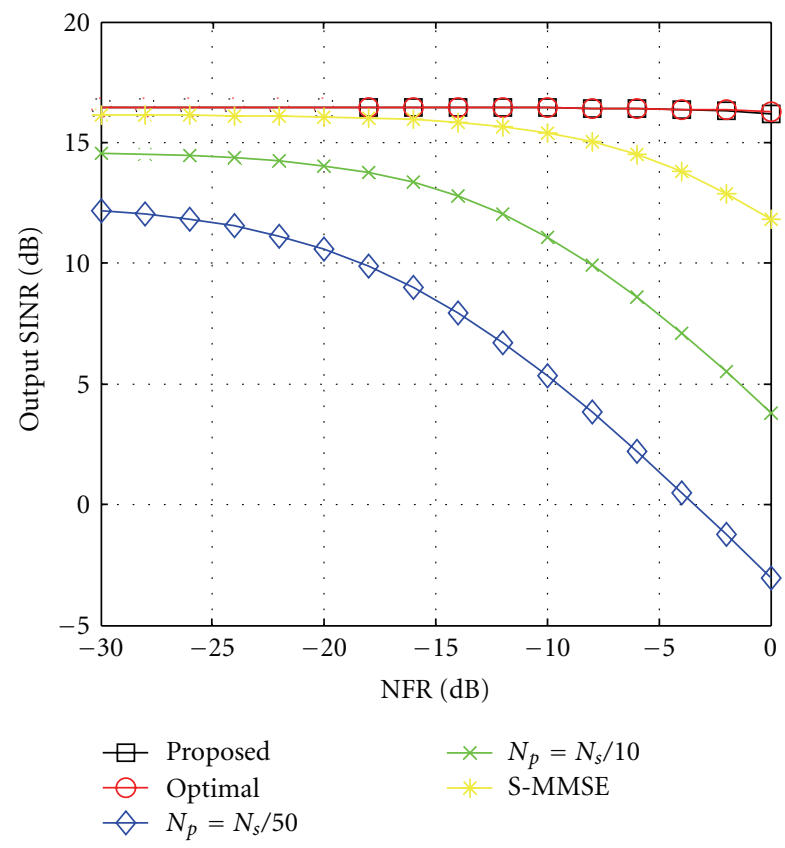

FIGURE 2: Output SINR performance versus NFR with $N=2, M=$ $32, L=4, K=10, \mathrm{SNR}=-10 \mathrm{~dB}$, and $N_{s}=500$.

In the fourth set of simulation, the BER performance was examined by varying input SNR with NFR $=10 \mathrm{~dB}$ and $N_{s}=500$. The results depicted in Figure 4 showed that the proposed receiver can possess a performance with a little degradation as the optimal one. It is noteworthy that the proposed scheme did not perfectly decode the transmit symbols in low-output SNR, leading to a slight inferiority to the optimal receiver. On the other hand, all the other 


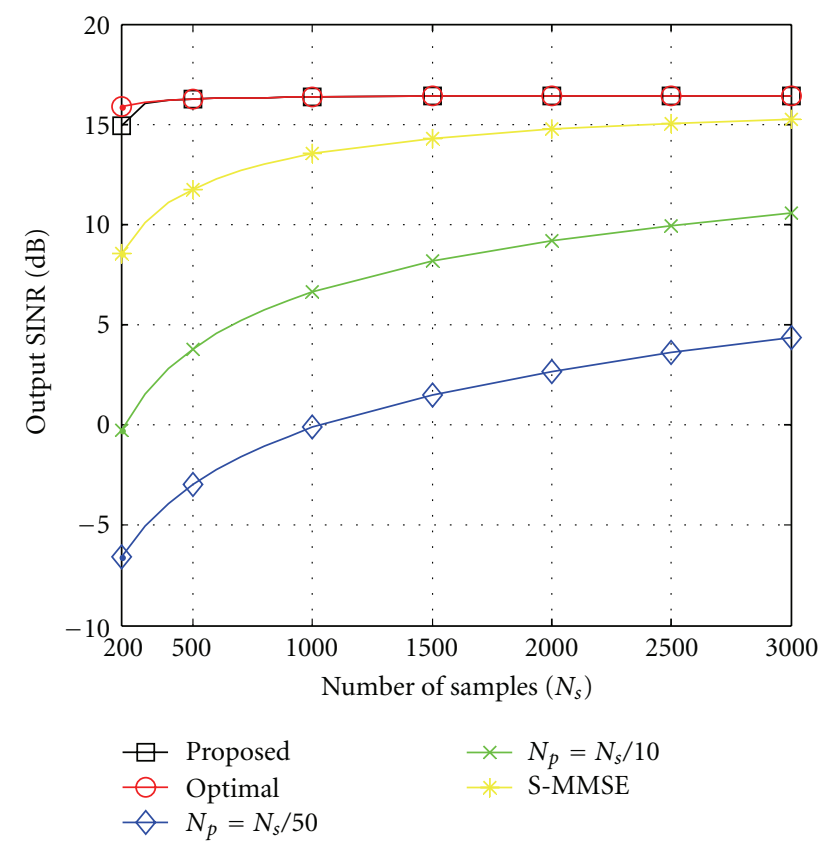

FIgURE 3: Output SINR performance versus sample size $N_{s}$ with $N=2, M=32, L=4, K=10, \mathrm{SNR}=-10 \mathrm{~dB}$, and $\mathrm{NFR}=10 \mathrm{~dB}$.

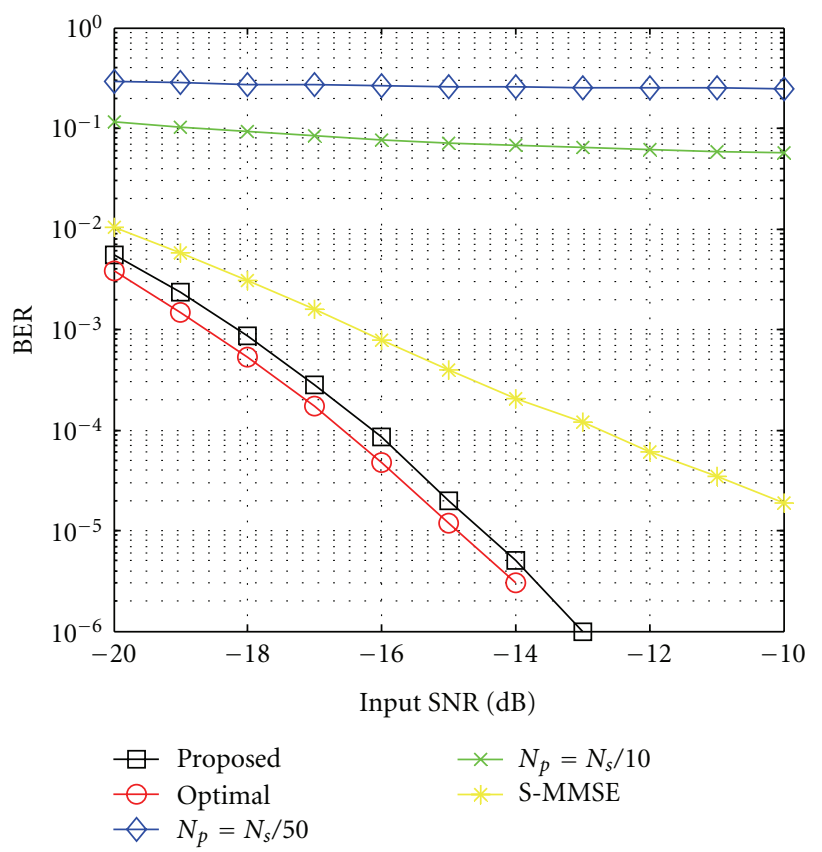

FIGURE 4: BER performance versus input SNR with $N=2, M=32$, $L=4, K=10, \mathrm{NFR}=10 \mathrm{~dB}$, and $N_{s}=500$.

receivers once again did not provide a reliable performance due to desired signal cancellation.

In the final set of simulations, the ISI effect was evaluated by setting the length of zero-padding equal to zero, that is, $N_{g}=0$, with NFR $=10 \mathrm{~dB}$ and $N_{s}=500$. The received data was reconstructed according to [16]. For comparison, the result obtained with the optimal receiver in an ISI-free case

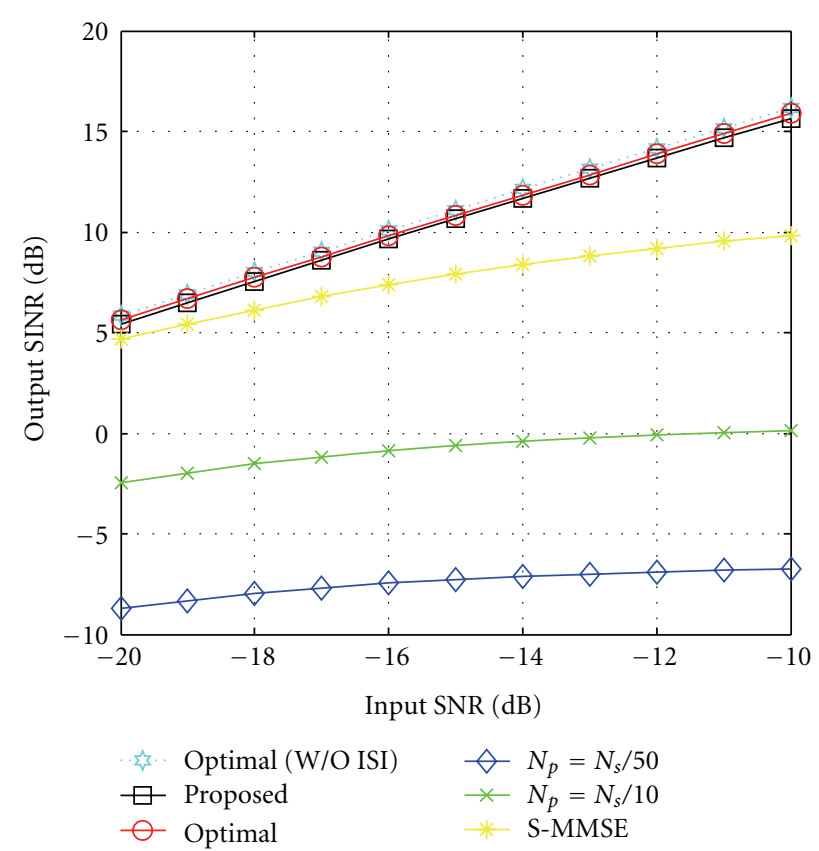

FIGURE 5: The ISI effect on the compared receivers when $N=2$, $M=32, L=4, K=10, \mathrm{NFR}=10 \mathrm{~dB}$, and $N_{s}=500$. The length of zero-padding is zero.

was also included, denoted by "Optimal (W/O ISI).” In order to combat the ISI, the $(3 \times 2 K-1)$ largest eigenvectors in (18) were chosen to determine the IB transformation. Figure 5 showed the curve of output SINR versus input SNR. It is observed output SINR increased with the input SNR, confirming the efficacy of the proposed two-stage receiver to combat both the MAI and ISI. As compared with the ISI-free case, the proposed two-stage receiver performed with a slight degradation about 0.51 and $0.44 \mathrm{~dB}$ for input SNR equal to -20 and $-10 \mathrm{~dB}$, respectively. This is because that the more degree of freedom was required to suppress ISI and resulted in noise enhancement.

\section{Conclusion}

In this paper, a blind two-stage MIMO receiver with enhanced MAI suppression has been proposed. Numerical results demonstrate that the proposed scheme without requirement of pilot symbols can effectively improve the robustness against the finite data sample effect and can provide a similar performance as to the fully data-aided MMSE (optimal) receiver.

\section{Acknowledgments}

This paper was sponsored jointly by the National Science Council, Taiwan, under Grant NC-99-2221-E-239-021. This paper was presented in part at the 2008 International Workshop on Antenna Technology on Small Antennas and Novel Metamaterials, Chiba, Japan, March 2008. 


\section{References}

[1] T. S. Rappaport, Wireless Communications, Principles and Practice, Prentice Hall, NJ, USA, 2002.

[2] H. Li, X. Lu, and G. B. Giannakis, "Capon multiuser receiver for CDMA systems with space-time coding," IEEE Transactions on Signal Processing, vol. 50, no. 5, pp. 1193-1204, 2002.

[3] S. M. Alamouti, "A simple transmit diversity technique for wireless communications," IEEE Journal on Selected Areas in Communications, vol. 16, no. 8, pp. 1451-1458, 1998.

[4] J. L. Yu and I. T. Lee, "MIMO capon receiver and channel estimation for space-time coded CDMA systems," IEEE Transactions on Wireless Communications, vol. 5, no. 11, pp. 3023 3028, 2006.

[5] H. Andoh, M. Sawahashi, and F. Adachi, "Channel estimation filter using time-multiplexed pilot channel for coherent RAKE combining in DS-CDMA mobile radio," IEICE Transactions on Communications, vol. E81-B, no. 7, pp. 1517-1525, 1998.

[6] T. T. Lin, "Performance analysis of non-data aided SBIB receivers for CDMA systems," Signal Processing, vol. 88, no. 6, pp. 1340-1354, 2008.

[7] J. Scott Goldstein and I. S. Reed, "Reduced-rank adaptive filtering," IEEE Transactions on Signal Processing, vol. 45, no. 2, pp. 492-496, 1997.

[8] J. L. Yu, "A low-complexity two-stage receiver for space-time block-coded CDMA MIMO systems," Signal Processing, vol. 87, no. 7, pp. 1626-1641, 2007.

[9] J. Namgoong, T. F. Wong, and J. S. Lehnert, "Subspace multiuser detection for multicarrier DS-CDMA," IEEE Transactions on Communications, vol. 48, no. 11, pp. 1897-1908, 2000.

[10] J. H. Mathews and R. W. Howell, Complex Analysis for Mathematics and Engineering, Jones and Bartlett, 5th edition, 2006.

[11] J. L. Yu and C. C. Yeh, "Generalized eigenspace-based beamformers," IEEE Transactions on Signal Processing, vol. 43, no. 11, pp. 2453-2461, 1995.

[12] B. W. Parkinson, J. J. Spilker Jr., P. Axelrad, and P. Enge, Global Positioning Systems: Theory and Applications, American Institute of Aeronautics and Astronautics, 1994.

[13] S. Glisic, Advanced Wireless Communications: 4G Technologies, John Wiley \& Sons, 2004.

[14] M. G. Di Benedetto and G. Giancola, Understanding Ultra Wide Band: Radio Fundamentals, Prentice Hall, Upper Saddle River, NJ, USA, 2004.

[15] T. T. Lin, "A blind anti-jammer pre-processor for GPS receiver," IEICE Transactions on Communications, vol. E88-B, no. 5, pp. 2215-2219, 2005.

[16] E. Aktas and U. Mitra, "Complexity reduction in subspacebased blind channel identification for DS/CDMA systems," IEEE Transactions on Communications, vol. 48, no. 8, pp. 13921404, 2000. 

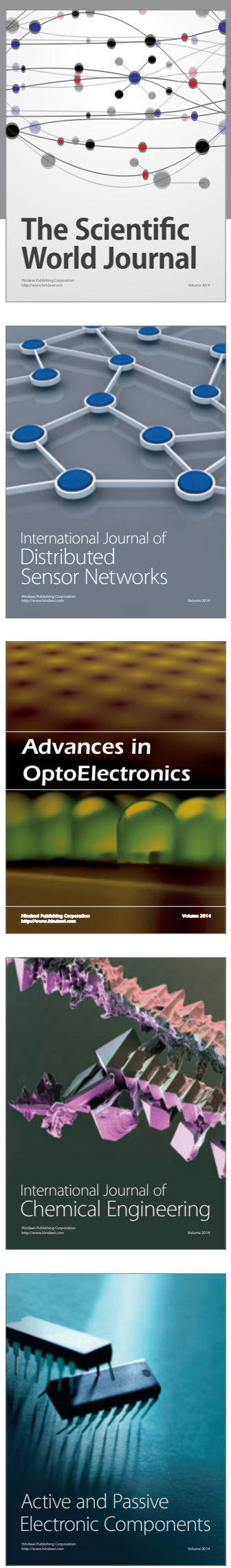
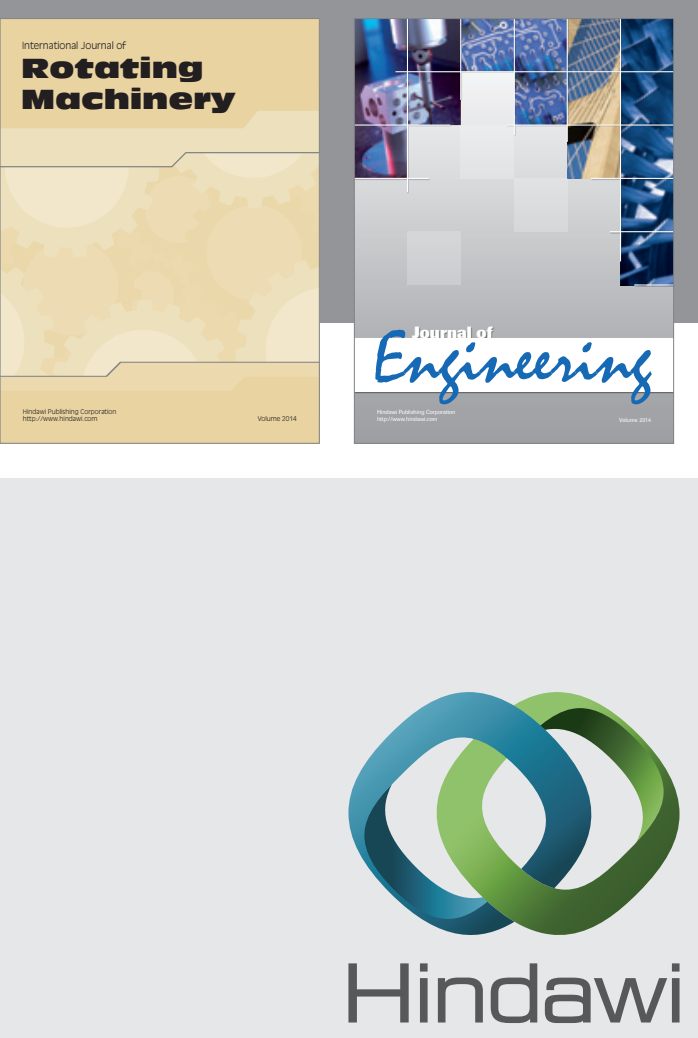

Submit your manuscripts at

http://www.hindawi.com
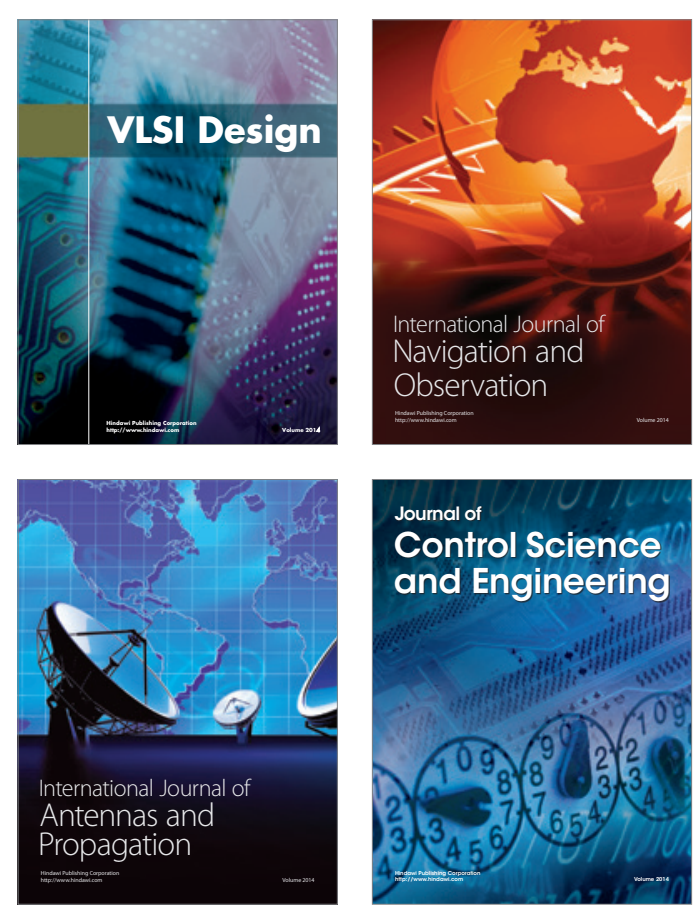
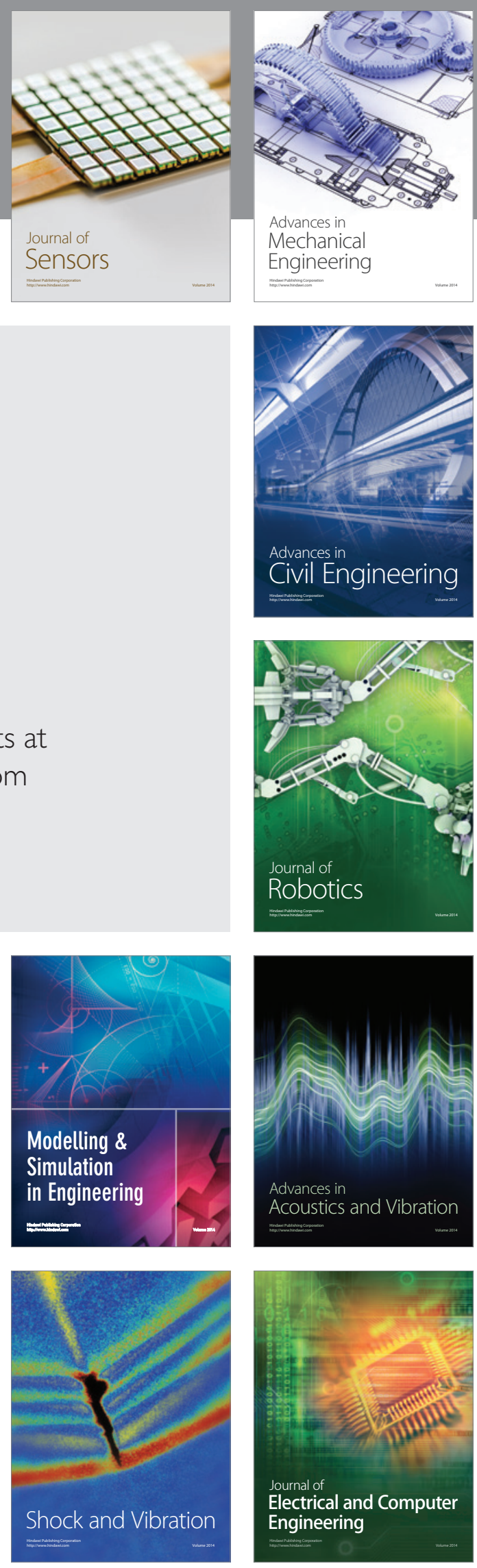